as abortion storms and 100\% mortality in newborn livestock, resulting in trade-bans which devastates the local and national economy. RVF is transmitted by direct contact with infected livestock and through mosquito bites, and has potential for global expansion. RVF has the "One Health" dimension where humans, animals, and the environment interact in spreading the disease. This needs special strategy to communicate the risk of RVF during outbreaks.

Methods: A cross-sectional community-based study was conducted in Sudan in 2013. A special One Health questionnaire was developed to compile data from 235 households. Face-toface interviews were conducted in an area that was exposed to the RVF outbreak.

Results: The community practiced risk factors such as handling sick animals, helping animals to deliver without protection measures, was only partially using mosquito bed nets, and usually not impregnated. Information about the RVF outbreak was mainly gained through social networks, while the health system or veterinarians were not used as an information source. This increases the possibility of rumors, wrong information and consequently disease dispersal.

Conclusion: We found that formulating the One Health approach team from different disciplines would be the best strategy to communicate the countermeasures to control RVF zoonotic outbreak. This is particularly useful when resources are limited and resilience is needed. The communication should consider the social cultural practices of the community, and highlight the different dimensions of zoonotic transmission to avoid spread of further RVF outbreak.

Prehosp Disaster Med 2017;32(Suppl. 1):s190-s191

doi:10.1017/S1049023X17005027

\section{Innovative Technological Approaches for Community}

Resilience

Sakiko Kanbara ${ }^{1}$, Limor Aharonson-Daniel' ${ }^{2}$, Hiroyuki Miyazaki ${ }^{3}$,Odeya Cohen ${ }^{2}$, Odeda Benin-Goren ${ }^{2}$, Dror Yifrah ${ }^{2}$, Ayumi Arai

1. Graduate School of Nursing, University of Kochi, Kochi/Japan

2. Ben-Gurion University of Negev, Beer-Sheva/Israel

3. University of Tokyo, Tokyo/Japan

Study/Objective: This proposal is a practical, solutionoriented research which, in a way, challenges the conventional public health surveillance systems - which require real-time or near real-time, population-based, statistical alarms to alert to unusual activity - through innovative ongoing surveillance that will incorporate geospatial assessment as well as behavioral and self-reported information.

Background: The Conjoint Community Resilience Assessment Measure (CCRAM) was developed by a multidisciplinary group of Israeli experts in order to offer a standard tool that will provide reliable information that can be useful when attempting to maintain or enhance community resilience.

Methods: Frequent and broadly distributed data collection using the CCRAM, on a cellular device, will be utilized for establishing and continuously supporting a society that is more resilient and literate in the field of disaster risk reduction. Smartphones and Internet cloud services will be used for data collection and management. The research program comprises of both System development of the mobile and cloud service and Experimental operation of the developed system, with Information and Communication Technology (ICT) trainings for community participants and health care providers.

Results: One of the added values of the project is to develop the next methodological model of health monitoring, offering a strong interactive network which crosses cultural and societal differences, geographies, and generations.

Conclusion: The potential of this research is not only to produce innovative research outcomes involving the improvement or optimization of services utilizing ICT, but also to promote research knowledge and idea exchange regarding social issues and challenges in the field of emergency preparedness and response.

Prehosp Disaster Med 2017;32(Suppl. 1):s191

doi:10.1017/S1049023X17005039

\section{Barriers and Opportunities for Early Detection of Breast Cancer in Gaza Rola Shaheen \\ Radiology, Peterborough Regional Health Center, Peterborough/ON/ Canada}

Study/Objective: Assessment of the landscape of barriers and opportunities of early detection of breast cancer, including women's and healthcare providers' awareness, knowledge, attitude, practice and access to breast health care in Gaza.

Background: Breast cancer is the most common malignancy and leading cause of cancer mortality among females in Gaza. Most cases are diagnosed at late stages. Survival rates are persistently low in contrast to improved rates worldwide.

Methods: In May and June 2014, using convenience and representative sampling, 100 healthcare providers completed surveys on knowledge of breast cancer, attitudes and practices. Structured interviews conducted for 100 women, 30 years and older across all districts and socio-economic backgrounds. Women were interviewed for knowledge of breast cancer, self-exam, attitude and practice when a breast problem encountered, access, and barriers. Data was analyzed using excel to find frequency distributions.

Results: Healthcare providers surveys: Only 15\% offer breast exam to their patients, $13 \%$ believe mammography can cause cancer, $48 \%$ do not know that radiotherapy is not available in Gaza, and $4 \%$ believe breast cancer is contagious. About 59\% of healthcare providers believe breast cancer is a fatal disease. Almost half of healthcare providers do not get opportunities to attend national or international medical conferences addressing breast cancer; and only $8 \%$ agreed for the need for accreditation with quality assurance program. Only $25 \%$ of surveyed women reported practice of self-examination, $87 \%$ had never had a mammogram or had been offered a breast examination, however $80 \%$ agreed to seeking a breast work-up when needed.

Conclusion: Knowledge gaps in breast health awareness and practice exist among healthcare providers and women in Gaza, with several identified opportunities for improvement. Most surveyed 
women never had a mammogram or clinical breast examination, however they are willing to have a breast work-up when needed.

Prehosp Disaster Med 2017;32(Suppl. 1):s191-s192

doi:10.1017/S1049023X17005040

\section{Prehospital Bio-event Detection: An Assessment of} Syndromic Surveillance Systems in Australian Ambulance

Services

\section{Cameron Anderson}

School of Medical and Health Sciences, Edith Cowan University, Joondalup/WA/Australia

Study/Objective: The objective of this research is to assess disease surveillance methods, used by Australian ambulance services, and provide a current picture of Australia's prehospital disease surveillance capability.

Background: The threat of bio-events, such as disease or bioterrorism, requires innovative surveillance methods to rapidly recognize novel and obscure threats, permitting early implementation of measures to limit the spread of disease. Ambulance call and dispatch data are enticing, due to their immediacy, geographic specificity, and reach into the community. However, implementing the data into functioning surveillance systems has proven problematic, due to the broad, non-specific nature of ambulance call categorization.

Methods: Each of the eight emergency ambulance services in Australia were invited to participate in an interview to establish the history, utility, and learnings from the use of call data for disease surveillance. Qualitative analysis sought to identify common issues and themes across the country.

Results: One Australian ambulance service, the Ambulance Service of New South Wales, uses its data for background surveillance within a surveillance system run by the New South Wales Ministry of Health, which encompasses several different data sources. All ambulance services participating in this research have identified the ability to undertake active surveillance during known emergencies. However, many inconsistencies were noted as to whether screening should be implemented, which callers should be screened, and what questions should be asked.

Conclusion: The potential for real-time, ambulance-based disease surveillance in Australia exists. However, at this point in time, none of the Australian ambulance services involved in this research currently conduct real-time surveillance. With one exception, no services routinely perform surveillance at all; nor were any plans identified to start doing so. All services have the ability to undertake disease screening during known emergencies; but how can these be made more consistent and reliable as an "all-hazards" early warning system?

Prehosp Disaster Med 2017;32(Suppl. 1):s192

doi:10.1017/S1049023X17005052

The Burden of Matatu Bus Crashes in Kenya Isaac M. Botchey Jr ${ }^{1}$, Yuen W. Hung ${ }^{1}$, Amber Mehmood ${ }^{1}$, Abdulgafoor Bachani ${ }^{1}$, Saidi Hassan ${ }^{2}$, Adnan A. Hyder ${ }^{1}$

1. Johns Hopkins International Injury Research Unit, Johns Hopkins Bloomberg School of Public Health, Baltimore/MD/United States of America
2. Department Of Anatomy, School Of Medicine, University of Nairobi, Nairobi/Kenya

Study/Objective: To describe the burden of Matatu Crashes in Kenya using multi-site injury surveillance data.

Background: Road Traffic Crashes (RTCs) are estimated to cause 1.3 million deaths worldwide each year. In Kenya, this problem is particularly significant and matatus are thought to be frequently involved. Matatus are 14-seater mini-buses responsible for transporting 12 million commuters daily in Kenya.

Methods: Electronic-based trauma registries were established at five referral hospitals in Kenya: Kenyatta National, Thika Level 5, Naivasha District, Machakos Level 5 and Meru Level 5. Information on the mechanism of injury, injury severity, patient outcomes, and patterns of care (prehospital and hospital-based) was collected. Results: The total number of presenting trauma patients was 24,014 . Road traffic injuries accounted for $41.7 \%$ of all presenting trauma patients. Matatus accounted for $20.4 \%$ of all RTCs. Despite the high occurrence, the injury severity of matatu crash victims was significantly less than other road traffic injuries $(\mathrm{p}<0.001)$. 68.7\% of matatu crash victims were discharged directly from the Accident \& Emergency Ward, which is significantly higher than the overall patient discharge rate $(\mathrm{p}<0.001)$. Of admitted patients, $56.7 \%$ suffered from a lower extremity injury. Seat belt use was significantly lower among matatu crash victims, when compared to other RTCs where seatbelt use was possible $(\mathrm{p}<0.001)$.

Conclusion: Matatus are already highly regulated and continue to be the focus of many road safety policies in Kenya. Accident \& Emergency wards are burdened with minor injuries of matatu victims, predominantly involving lower extremities. Safe road practices among drivers and passengers can reduce this burden.

Prehosp Disaster Med 2017;32(Suppl. 1):s192

doi:10.1017/S1049023X17005064

Influenza-Like Illness and Gastrointestinal Illness: Surveillance Using a Novel Online Bio Surveillance System in Child Care Centers Sarab Ewing ${ }^{1}$, Jim Cranford ${ }^{2}$, Andrew N. Hashikawa ${ }^{2}$

1. University of Michigan Medical School, Ann Arbor/United States of America

2. Department Of Emergency Medicine, University of Michigan, Ann Arbor/MI/United States of America

Study/Objective: To describe the capability of a novel, online, child care center bio surveillance system (sickchildcare.org) to report pediatric Influenza-Like Illness (ILI) and Gastrointestinal (GI) illness outbreaks compared to the state surveillance system.

Background: Bio surveillance is critical for early detection of disease outbreaks and resource mobilization. Children in child care centers are frequently sick and first to become ill. We created a free, web-based surveillance system (sickcbildcare.org - SCC) for child care centers to report sick children. In comparison, the state's surveillance system (Michigan Care Improvement Registry (MCIR)) uses traditional grade school and hospital system reports. Data from sickchildcare.org has not been compared to the state's surveillance system. 